\title{
Sucrose Density Gradient Centrifugation Separation of Gold and Silver Nanoparticles Synthesized Using Magnolia kobus Plant Leaf Extracts
}

\author{
So Hyun Lee, Bipinchandra K. Salunke, and Beom Soo Kim
}

Received: 27 August 2013 / Revised: 15 January 2014 / Accepted: 19 January 2014

(C) The Korean Society for Biotechnology and Bioengineering and Springer 2014

In the 2014 issue of Biotechnology and Bioprocess Engineering (BBE), an error occurred in the research article: Youl Her, Young-Chul Lee, Jin-Hwan Oh, Yoon-E Choi, Chang-Woo Lee, Jin-Suk Kim, Hwan Mook Kim, and Ji-
Won Yang (2014) An Application of $\beta$-glycosidase to Transformation of Ginsenosides for the Effective Production of Specific Ginsenosides with Biological Efficacy. Biotechnol. Bioprocess Eng. 19: 169-174.

- In the Fig. 1

Original:
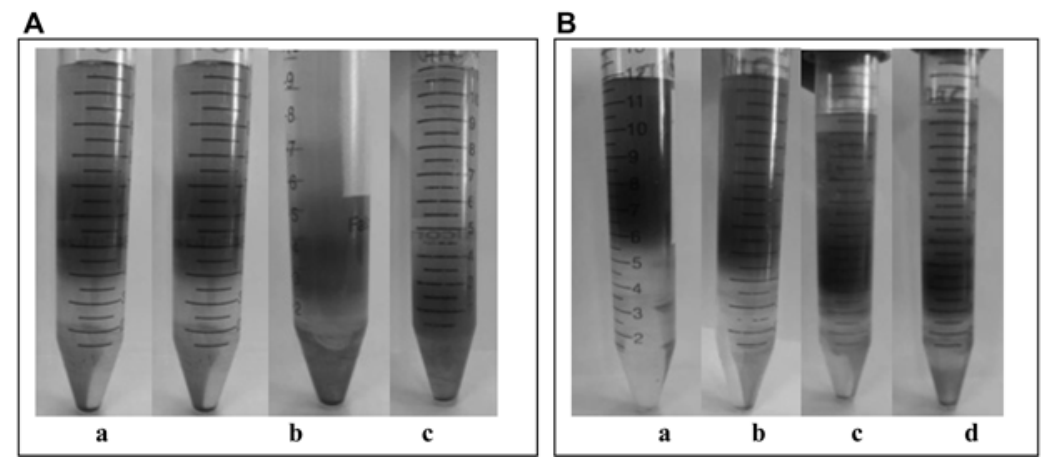

This should be replaced by:
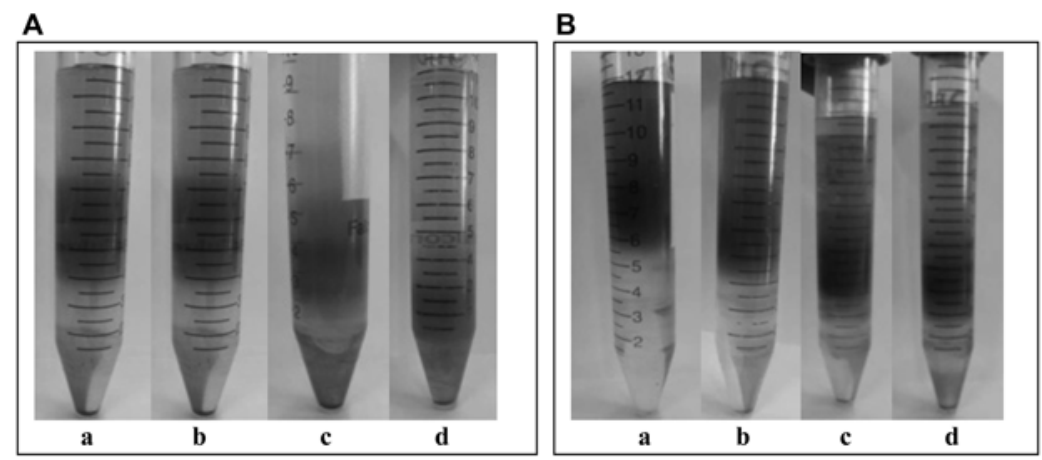

Received: 10 March 2014

So Hyun Lee, Bipinchandra K. Salunke, Beom Soo Kim*

Department of Chemical Engineering, Chungbuk National University,

Cheongju 361-763, Korea

Tel: +82-43-261-2372; Fax: +82-43-269-2370

E-mail: bskim@chungbuk.ac.kr 